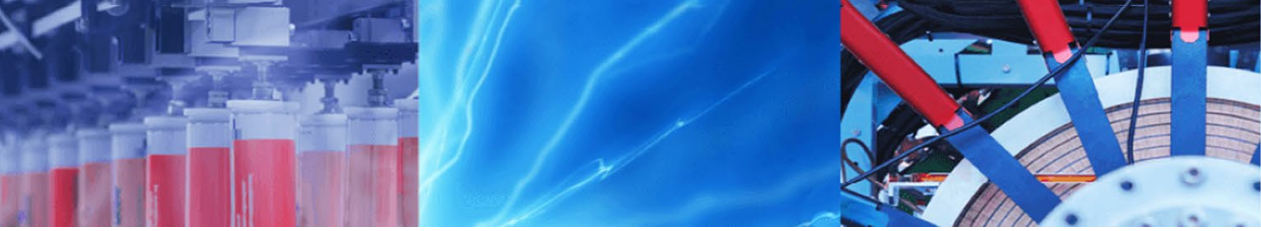

Research Article

\title{
Improved prediction method for ground surface thawing settlement caused by the melting of tunnel horizontal frozen wall
}

\author{
Rongbao Hong ${ }^{1,2} \cdot$ Haibing Cai ${ }^{1,2} \cdot$ Mengkai $\mathrm{Li}^{1,2}$
}

Received: 17 June 2021 / Accepted: 27 August 2021

Published online: 08 September 2021

(c) The Author(s) 2021 OPEN

\begin{abstract}
With the rapid development of urban subway tunnel, artificial ground freezing technology is becoming more and more mature. With the natural thawing of horizontal frozen wall, thawing settlement will occur on stratum due to the thawing of frozen soil and the consolidation of thawed soil, which will inevitably bring adverse impact on the surrounding environment of subway tunnel. Therefore, the establishment of a reasonable ground surface thawing settlement prediction method will provide a favorable theoretical support for predicting the ground surface deformation in advance and taking active thawing settlement control measures. In the paper, the time functions of ground surface thawing settlement and consolidation settlement of tunnel horizontal frozen wall are established based on the stochastic medium theory during natural thawing period, and the calculation methods of thawing front radius, inner radius of thawing shrinkage region and inner radius of consolidation region are proposed. The results show that the cumulative ground surface thawing settlement is larger than that of Cai et al. after considering the consolidation of the thawed soil, which fully indicates that the ground surface settlement caused by the drainage and consolidation of the thawed soil cannot be ignored. In addition, the thawing displacement rate of frozen soil is greater than the consolidation displacement rate of thawed soil during the natural thawing and the thawed soil will be consolidated at a lower settlement rate for a long time after the natural thawing period.
\end{abstract}

Keywords Artificial ground freezing technology · Horizontal frozen wall · Thawing settlement · Consolidation · Stochastic medium theory

\section{Introduction}

The idea of artificial freezing originates from seasonal ground freezing under natural conditions. Artificial frozen soil has high bearing capacity and strong water sealing capacity, which fully meets the requirements of temporary ground support in underground structure construction. Therefore, artificial ground freezing technology has been rapidly adopted by many countries and applied to the construction of coal mine shaft [1]. Meanwhile, with the acceleration of urbanization, in order to alleviate the pressure of land traffic, people gradually turn their attention to the development of urban underground space. In the 1990s, artificial horizontal freezing reinforcement technology was successfully applied in many tunnel construction sections of Shanghai Metro line 1, line 2 and Beijing Metro "Fu-Ba" line [2]. In the past 20 years, China's coastal cities such as Guangzhou, Shenzhen, Nanjing, Suzhou and Nantong have constantly broken the record of artificial horizontal freezing length, making China's artificial

Rongbao Hong, cherishrb2020@163.com; Haibing Cai, haibingcai@163.com; Mengkai Li, mengkaili2020@163.com | 'School of Civil Engineering and Architecture, Anhui University of Science and Technology, Huainan 232001, China. ${ }^{2}$ Engineering Research Center of Underground Mine Construction, Ministry of Education, Huainan 232001, Anhui, China.

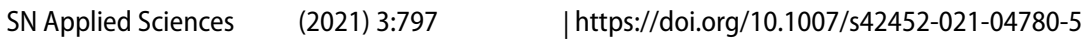


freezing technology leap to the world's leading level [3]. However, the thawing settlement caused by the melting of horizontal frozen wall in the subway tunnel construction has always been the focus of the engineering research.

During the natural thawing of horizontal frozen wall, the temperature of frozen soil rises continuously, the ice crystals in frozen soil gradually thaw, and its volume reduces. At the same time, under the joint action of overlying soil and surface surcharge, the soil consolidates and compresses. The resulting comprehensive effect is thawing settlement [4]. Research shows that the volume expansion of soil during freezing is about $9 \%$ [5]. Therefore, the thawing settlement of frozen soil during natural thawing of freezing wall is considered as the reverse process of soil volume expansion, which is generally characterized by thawing settlement coefficient [6]. At present, in the study of consolidation and compaction settlement of thawed soil, the laboratory test only uses the compaction coefficient [7] to characterize the macro-phenomenon and does not consider the water migration in the thawed soil. In 1925, Terzaghi put forward the principle of effective stress, established Terzaghi's theory of saturated soil consolidation and quantified the calculation method of soil consolidation settlement. He thinks that the soil can be compressed and deformed in one direction, and the water in the soil flows in one direction according to Darcy's law. Then, Nixon [8] applied the theory to the thawing compression test of artificial frozen soil, combined with the power function of thawing front changing with time, established the time-dependent function of effective stress in thawed soil and calculated the consolidation settlement displacement of thawed soil. The thawing of artificial horizontal frozen wall can be considered as a twodimensional problem [9], as shown in Fig. 1, so the soil consolidation theory still has a certain applicability in the study of this problem.

In addition, there are many methods predicting the ground surface settlement, such as artificial neural network

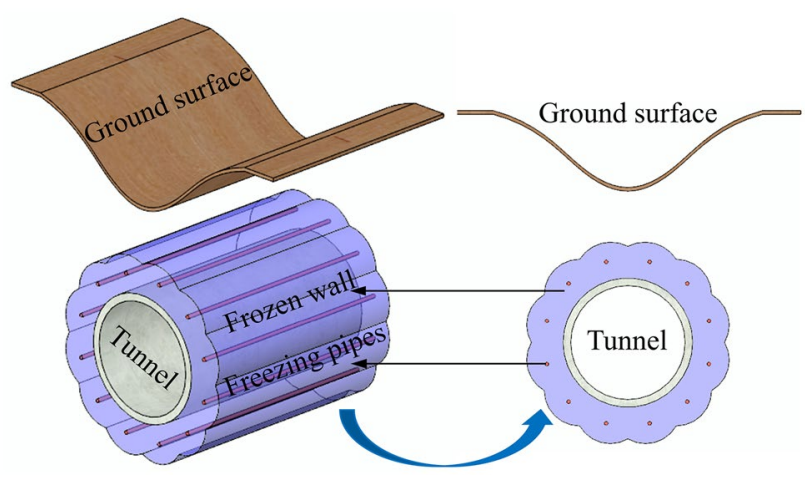

Fig. 1 Simplification of thawing model method [10], artificial bee colony algorithm [11], Peck method [12] and stochastic medium theory [13]. Artificial neural network method and artificial bee colony algorithm are more inclined to evaluate the risk of ground surface collapse, while Peck method is put forward based on the concept of soil loss. Obviously, stochastic medium theory is more suitable for the prediction of ground surface thawing settlement because the thawing settlement process of artificial horizontal freezing wall is regarded as a random process. Yang et al. [14] analyzed the surface movement and deformation caused by several tunnel construction methods, such as precipitation method, freezing method, extrusion shield method, compressed air method, and proposed a feasible prediction method of surface settlement based on the stochastic medium theory. Ning [15] predicted the ground thawing settlement caused by freezing construction of a side passage of Shanghai Metro pearl line, and compared it with the measured deformation data. The predicted vertical surface deformation curve is consistent with the measured data curve, which fully proves the practicability of the stochastic medium theory ground thawing settlement prediction model. Liu et al. [16] combined the soil pressure and thawing settlement coefficient with the stochastic medium theory to predict the surface settlement caused by the freezing method construction of a subway tunnel side passage, and drew a conclusion with reliable engineering application value. Shi et al. [17] used stochastic medium theory and soil consolidation compaction theory to analyze the settlement and deformation of the stratum in the dewatering stage of dewatering well before tunnel construction, and concluded that the closer the dewatering well is to the tunnel, the greater the settlement value of the stratum. Furthermore, Shi et al. [18] used the same analysis method to predict the settlement, inclination and deformation of soil in the disturbed area of tunnel pipe jacking construction, which provided guarantee for the formulation of effective settlement control measures at the construction site. Cai et al. [9, 19-23] introduced the power function of thawing front changing with time in the study of thawing process of horizontal frozen wall, assumed that the natural thawing transient temperature field of frozen wall conforms to the plate thawing theory, and the forced thawing conforms to the single tube thawing theory, and improved the calculation method of average temperature of frozen wall under forced thawing. Taking the stable settlement of onedimensional frozen soil melting as the upper and lower limits of double integral of stochastic medium theory, a diachronic prediction model of surface settlement is established. The model has good response to the thickness of frozen wall, tangent value of soil main influence angle, tunnel depth, melting and compaction coefficient, and can reflect the real process of surface settlement more accurately. Wu [24] thinks that the displacement of the bottom soil is not zero in the 
convergence process of the tunnel excavation section, and verifies it by using the stochastic medium theory. It is found that the convergence law of the tunnel excavation section obtained by this convergence model is more practical than that obtained by the uniform convergence model. Li et al. [25] assumed that the soil loss at the variable cross section of the tunnel is linear, and established the calculation model of the surface settlement and horizontal displacement at the variable cross section of the tunnel through the stochastic medium theory. The calculation results show that the slope of the surface settlement curve at the variable cross section increases with the increase in the ratio of the tangent of the main influence angle of the stratum to the buried depth of the tunnel, and the application scope of stochastic medium theory in tunnel engineering is further expanded.

In the process of predicting the surface deformation of tunnel construction, the above stochastic medium theory has drawn some meaningful conclusions, but it has not analyzed the consolidation of thawed soil due to the self-weight stress of overlying soil layer and surface surcharge during the thawing period of tunnel horizontal frozen wall. In view of this, this paper further optimizes the ground surface settlement diachronic prediction model proposed by Cai et al. [9], aiming to solve the problem of conservative prediction of the original model. Besides, different from the modeling ideas of Cai et al., this model focuses on reflecting the phenomena of the thawing of frozen soil and consolidation of thawed soil during the melting of horizontal frozen wall.

Specifically, before the theoretical derivation, the thawing model is simplified, as shown in Fig. 1. This paper takes a two-dimensional section of horizontal frozen wall to study the ground surface settlement caused by the thawing of frozen soil and the consolidation of thawed soil. It combines the unidirectional consolidation theory of thawing soil with stochastic medium theory, so as to obtain the ground surface thawing settlement law more in line with the engineering practice. It will provide a theoretical calculation model for the ground surface thawing settlement during the tunnel construction using artificial ground freezing technology (AGF).

\section{Consolidation theory of soil}

\subsection{Average degree of consolidation}

Terzaghi studied the variation law of excess pore water pressure with time by exerting full pressure on saturated clay layer, established the basic differential equation of one-way consolidation and obtained the analytical solution under certain initial and boundary conditions, which has been used up to now. It is assumed that only one-dimensional vertical consolidation occurs in the soil in the melting zone.
After the beginning of drainage consolidation, the pore water pressure at the same point at different times is different, and at the same time, the pore water pressure at different positions of the stratum is also different. Therefore, the pore water pressure $(u)$ is a function of time $(t)$ and coordinate $(Z)$, namely $u=u(Z, t)$.

The basic differential equation of one-way consolidation established by Terzaghi is as follows:

$\frac{\partial u}{\partial t}=C_{v} \frac{\partial^{2} u}{\partial z^{2}}$

$C_{v}=\frac{k}{\gamma_{w}} \frac{1+e}{\alpha_{v}}$

$\alpha_{v}=\frac{e_{1}-e_{2}}{p_{1}-p_{2}}=\frac{\Delta e}{\Delta p}$

where $C_{v}$ is the consolidation coefficient of soil layer; $k$ is the permeability coefficient of soil layer; $\gamma_{w}$ is the volumetric weight of pore water; $a_{v}$ is the compressibility of soil layer; $e$ is the void ratio of the micro unit; $e_{1}$ is the void ratio under the condition of stress $p_{1}$ on the compression curve; $e_{2}$ is the void ratio under the condition of stress $p_{2}$ on the compression curve; $\Delta e$ is the change of soil void ratio; and $\Delta p$ is the change of soil stress.

Combining initial condition with boundary condition $\left(u(Z, 0)=u_{0}, u(0, t)=0, \partial u / \partial t(H, t)=0\right)$, Eq. (1) can be solved by the variable separation method; then, the particular solution is obtained by substituting the initial value condition $\left(u_{0}=\gamma_{w} Z\right)$.

$u(Z, t)=\sum_{m=1}^{\infty} \frac{8 \gamma_{w} h_{0}}{m^{2} \pi^{2}} \exp \left(-m^{2} \frac{\pi^{2}}{4} \cdot \frac{C_{v} t}{h_{0}^{2}}\right) \sin \left(\frac{m \pi}{2 h_{0}} Z\right)$

where $m$ is an odd positive integer $(1,3,5 \ldots \ldots.) ; u_{0}$ is the initial pore water pressure; and $h_{0}$ is the depth of precipitation. The meaning of other symbols is the same as before.

The stress of each point in the soil layer caused by drainage consolidation is not the same, and the consolidation degree of each point is also different. In practical application, the average degree of consolidation $\left(U_{t}\right)$ can be used to calculate.

$$
\begin{aligned}
U_{t} & =\frac{U_{0}-U(Z, t)}{U_{0}}=1-\frac{U_{0}}{U(Z, t)}=1-\frac{\int_{0}^{h_{0}} u(Z, t) d Z}{\int_{0}^{h_{0}} u_{0} d Z} \\
& =1-\frac{32}{\pi^{3}} \exp \left(-\frac{\pi^{2}}{4} \cdot \frac{C_{v} t}{h_{0}^{2}}\right)
\end{aligned}
$$




\subsection{Consolidation compaction function}

Figure 2a shows the one-dimensional drainage-consolidation model, which only reflects the change of the vertical water level and displacement; however, the two-dimensional drainage-consolidation model is considered for the thawing settlement during the thawing of tunnel horizontal frozen wall, as is shown in Fig. $2 \mathrm{~b}$. Through coordinate system transformation, the precipitation depth $\left(h_{0}\right)$ and initial water level depth $\left(H_{0}\right)$ can be transformed into:

$h_{0}=\left(R_{1}-R_{0}\right) \sin \theta$

$H_{0}=h-R_{1} \sin \theta$

The consolidation displacement component $\left(d s\left(\eta_{1}, t\right)\right)$ generated by the drainage consolidation of micro unit soil $(\mathrm{d} \varepsilon \mathrm{d} \eta)$ at time $t$ is shown as follows:

$d s\left(\eta_{1}, t\right)=\frac{\alpha_{v} \Delta p}{1+e_{0}} d \eta_{1}$

$\Delta p=U_{t} \gamma_{w}\left(\eta_{1}-H_{0}\right)$

where $e_{0}$ is the initial void ratio before consolidation and $\eta_{1}$ is the depth from the center of micro unit to the ground surface.

Then, the unit consolidation compaction function $G\left(\eta_{1}, \mathrm{t}\right)$ of the thawed soil is shown as follows:
$G\left(\eta_{1}, t\right)=\frac{d s\left(\eta_{1}, t\right)}{d \eta_{1}}=\frac{\alpha_{v} U_{t} \gamma_{w}\left(\eta_{1}-H_{0}\right)}{1+e_{0}}$

It can be considered that the micro unit soil has no lateral deformation after drainage consolidation, and the volume compression caused by drainage consolidation of the micro unit soil at the depth of $\eta_{1}$ is $G\left(\eta_{1}, t\right) \mathrm{d} \eta_{1}$.

\section{Diachronic prediction model of thawing settlement}

Before the horizontal frozen wall thaws naturally, the circular cylindrical frozen wall with outer radius of $R_{1}$ has been formed around the tunnel. In the general horizontal freezing project of tunnel, the soil temperature at the excavation boundary is negative. Therefore, the inside radius of frozen wall can be considered as $R_{0}$ (the outside radius of tunnel lining), and the effective thickness of the frozen wall is $R_{1}-R_{0}$. The inner edge radius of the frozen wall changes with the increase in thawing time ( $t$ ) due to the heat exchange between the frozen wall and the tunnel lining. Specifically, the radius of the thawing inner front is $R_{1}(t)$, the inner edge of the frozen wall gradually thaws from $R_{0}$ to $R_{a}(t)$, and then consolidation to $R_{b}(t)$. The inner thawing shrinkage region is $\Delta_{a}(t)$, and the inner consolidation region is $\Delta_{b}(t)$, as shown in Fig. 3a. The outer edge radius of the frozen wall changes with the increase in thawing time $(t)$ due to the heat exchange between the frozen wall and the external stratum. Specifically, the radius of the thawing outer front is $R_{2}(t)$, the outer edge of the frozen wall gradually thaws from $R_{1}$ to $R_{c}(t)$, and then consolidation to $R_{d}(t)$.
Fig. 2 Drainage-consolidation model: a one-dimensional; $\mathbf{b}$ two-dimensional

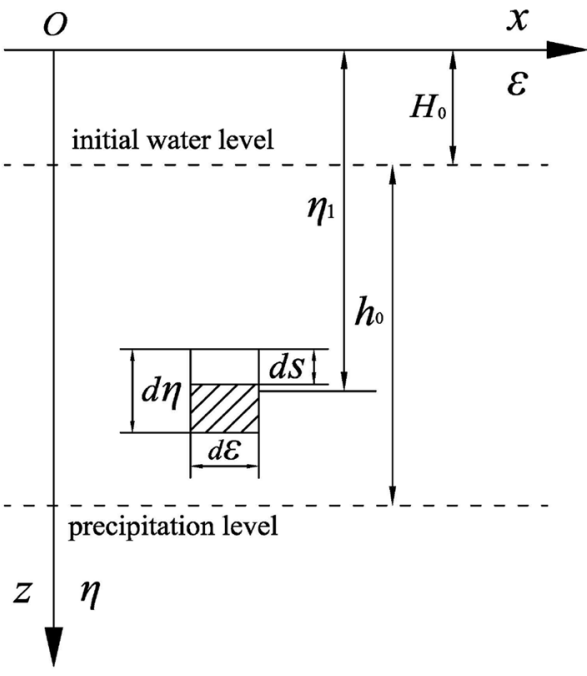

(a)

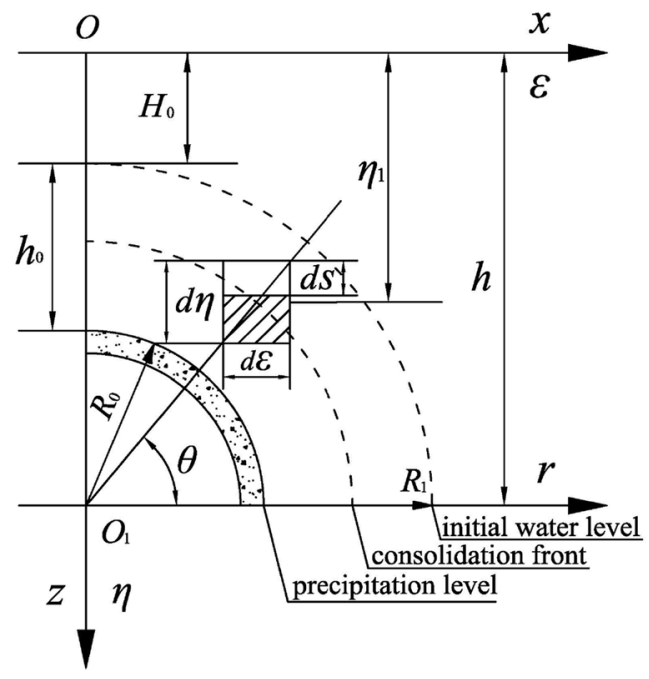

(b) 


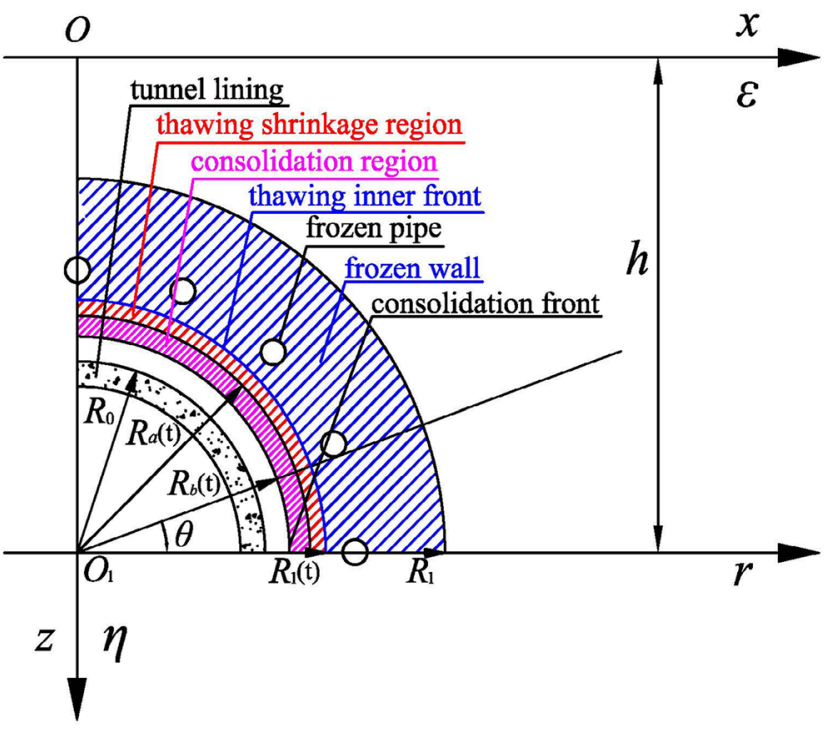

(a)

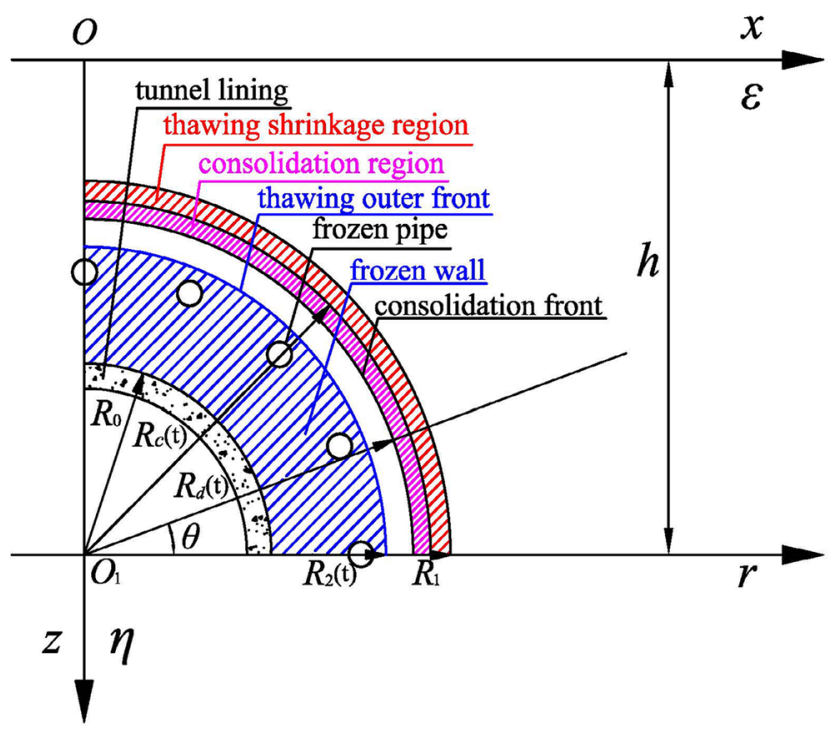

(b)

Fig. 3 Coordinate system transformation of frozen wall: $\mathbf{a}$ inner edge frozen wall; $\mathbf{b}$ outer edge frozen wall

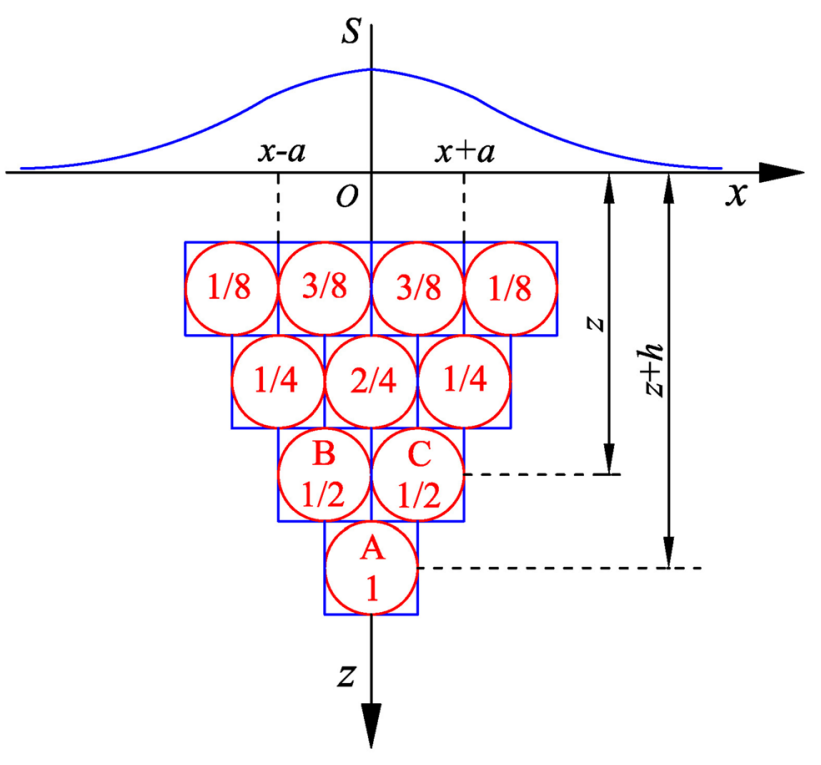

Fig. 4 Stochastic medium model

The outer thawing shrinkage region is $\Delta_{c}(\mathrm{t})$, and the outer consolidation region is $\Delta_{d}(t)$, as shown in Fig. $3 \mathrm{~b}$. In all, the accumulated thawing displacement and consolidation displacement of the stratum together reflect the thawing settlement of ground surface.

As can be seen from Fig. 4, when the ball $A$ is removed, ball $\mathrm{B}$ or $\mathrm{C}$ will fill its space with a certain probability. Correspondingly, the upper balls will fill the empty space downward in turn; therefore, the behavior can be described by the following probability expression. $\frac{1}{2} P(x-a, z)+\frac{1}{2} P(x+a, z)=P(x, z+h)$

Transforming the above equation by Lagrange mean value theorem, when $a$ and $h$ infinitely tend to zero, we can obtain the following differential equation.

$\frac{1}{2} \frac{\partial^{2} P(x, z)}{\partial x^{2}}=\frac{\partial P(x, z)}{\partial z}$

Giving Eq. (12) practical engineering meaning,

$B(z) \cdot \frac{\partial^{2} S(x, z)}{\partial x^{2}}=\frac{\partial S(x, z)}{\partial z}$

where $B(z)$ is the coefficient of soil heterogeneity and $S(x$, $z)$ is the vertical displacement.

According to the actual working conditions, the boundary condition can be expressed as:

$S(x, 0)=\delta(x)$

where $\delta(x)$ is the subsidence function of ground surface, which approximates a Gaussian curve.

Thus, the special solution of Eq. (13) is as follows.

$S_{e}(x, z)=\frac{1}{r(z)} \exp \left[-\frac{\pi}{r^{2}(z)} x^{2}\right]$

$r(z)=\frac{\eta-z}{\tan \beta}$

where $S_{e}(x, z)$ is the vertical settlement of micro unit with the coordinate of $(x, z), r(z)$ is the radius of the vertical 
settlement, $\eta$ is the depth from the center of the micro unit to the ground surface, and $\beta$ is the main influence angle of soil layer.

The main influence angle $(\beta)$ is related to the cohesion and the internal friction angle of soil layer. According to Terzaghi's loose earth pressure theory, the shear plane with the angle of $\left(45^{\circ}-\varphi / 2\right)$ along the horizontal direction is formed in the process of stress transfer of soil particles through mutual friction and sliding. Shi et al. [26] optimized the calculation method of the main influence angle combining with the retaining wall theory.

$\beta=90^{\circ}-\arctan \left[\tan \left(45^{\circ}+\frac{\varphi}{2}\right)+\frac{2 c}{\gamma H}\right]$

where $\varphi$ is the internal friction angle of soil; $c$ is the cohesion of soil; $\gamma$ is the volumetric weight of soil; and $H$ is the buried depth of the soil above the tunnel. For the sandy soil or saturated soft clay with very poor cohesion, that is $c=0 \mathrm{kPa}$, the main influence angle of the stratum degenerates to $\beta=45^{\circ}-\varphi / 2$.

Therefore, when $z=0$, the ground surface settlement caused by the thawing shrinkage and consolidation compaction of each micro unit in the thawing shrinkage region and consolidation region is $S_{1}(x)$ and $S_{2}(x)$, respectively, based on the stochastic medium theory, during the natural thawing process of horizontal frozen wall.

$S_{1}(x)=\frac{\tan \beta}{\eta} \exp \left[-\frac{\pi \tan ^{2} \beta}{\eta} x^{2}\right] d \varepsilon d \eta$

$S_{2}(x)=\frac{\tan \beta}{\eta} \exp \left[-\frac{\pi \tan ^{2} \beta}{\eta} x^{2}\right] G\left(\eta_{1}, t\right) d \varepsilon d \eta$

where $x$ is the abscissa of each point on the ground surface in the rectangular coordinate system ( $\varepsilon \circ \eta(x o z)) ; \mathrm{d} \varepsilon$ and $\mathrm{d} \eta$ are the length and width of micro unit.

For the plane problem, the calculation equation of the accumulated thawing settlement displacement caused by the natural thawing of the frozen wall during the horizontal freezing construction of the tunnel is as follows:

$S(x)=S_{1}(x)+S_{2}(x)$
Taking the rectangular coordinate system $\varepsilon o \eta(x O z)$ to the polar coordinate system $r o_{1} \theta$, the conversion equation is as follows:

$\varepsilon=r \cos \theta$

$\eta=h-r \sin \theta$

For the thawing of the inner edge of the horizontal frozen wall, the depth $\left(\eta_{1}\right)$ from the center of the micro unit to the ground surface at the consolidated front is as follows:

$\eta_{1}=h-R_{b}(t) \sin \theta$

For the thawing of the outer edge of the horizontal frozen wall, the depth $\left(\eta_{1}\right)$ from the center of the micro unit to the ground surface at the consolidated front is as follows:

$\eta_{1}=h-R_{d}(t) \sin \theta$

where $h$ is the distance between the tunnel center and the ground surface; $r$ and $\theta$ are the polar diameter and polar angle in the polar coordinate system $(r o, \theta)$.

The inner radius of the thawing shrinkage region $\left(\Delta_{a}(t)\right)$ of inner edge of frozen wall is $R_{a}(t)$, and the outer radius is $R_{1}(t)$. The inner radius of the consolidation region $\left(\Delta_{b}(t)\right)$ is $R_{b}(t)$, and the outer radius is $R_{a}(t)$. The inner radius of the thawing shrinkage region $\left(\Delta_{c}(t)\right)$ of outer edge of frozen wall is $R_{c}(t)$, and the outer radius is $R_{1}$. The inner radius of the consolidation region $\left(\Delta_{d}(t)\right)$ is $R_{d}(t)$, and the outer radius is $R_{c}(t)$. According to the exchange equation of double integral, the expressions of thawing displacement and consolidation displacement caused by natural thawing of frozen wall at time $(t)$ are as follows:

$$
\begin{aligned}
& S_{1}(x)=\iint_{\Delta_{a}(t)+\Delta_{c}(t)} \frac{\tan \beta}{h-r \sin \theta} \exp \left\{-\frac{\pi \tan ^{2} \beta}{(h-r \sin \theta)^{2}}[x-r \cos \theta]^{2}\right\} r d r d \theta \\
& =\int_{R_{a}(t)}^{R_{1}(t)} \int_{0}^{2 \pi} \frac{\tan \beta}{h-r \sin \theta} \exp \left\{-\frac{\pi \tan ^{2} \beta}{(h-r \sin \theta)^{2}}[x-r \cos \theta]^{2}\right\} r d r d \theta \\
& +\int_{R_{c}(t)}^{R_{1}} \int_{0}^{2 \pi} \frac{\tan \beta}{h-r \sin \theta} \exp \left\{-\frac{\pi \tan ^{2} \beta}{(h-r \sin \theta)^{2}}[x-r \cos \theta]^{2}\right\} r d r d \theta
\end{aligned}
$$

$$
\begin{aligned}
& S_{2}(x)=\iint_{\Delta_{b}(t)+\Delta_{d}(t)} \frac{\tan \beta}{h-r \sin \theta} \exp \left\{-\frac{\pi \tan ^{2} \beta}{(h-r \sin \theta)^{2}}[x-r \cos \theta]^{2}\right\} G\left(\eta_{1}, t\right) r d r d \theta \\
& =\int_{R_{b}(t)}^{R_{a}(t)} \int_{0}^{2 \pi} \frac{\tan \beta}{h-r \sin \theta} \exp \left\{-\frac{\pi \tan ^{2} \beta}{(h-r \sin \theta)^{2}}[x-r \cos \theta]^{2}\right\} G\left(h-R_{b}(t) \sin \theta, t\right) r d r d \theta \\
& +\int_{R_{d}(t)}^{R_{c}(t)} \int_{0}^{2 \pi} \frac{\tan \beta}{h-r \sin \theta} \exp \left\{-\frac{\pi \tan ^{2} \beta}{(h-r \sin \theta)^{2}}[x-r \cos \theta]^{2}\right\} G\left(h-R_{d}(t) \sin \theta, t\right) r d r d \theta
\end{aligned}
$$




\section{Determination of thawing front radius}

The solution of the inner and outer front radius $\left(R_{1}(t)\right.$ and $\left.R_{2}(t)\right)$ is the key to establish the prediction model of thawing settlement under natural thawing of horizontal frozen wall. Cai et al. [9] solved the transient temperature field of horizontal frozen wall approximately using plate thawing theory, and then obtained the calculation equations of the inner and outer front radius during thawing.

As shown in Fig. 5, assuming the thickness of the frozen wall is infinite, the tunnel lining and the unfrozen soil are thin plates, whose surface have the constant positive temperature $\left(T_{b}\right)$; correspondingly, the soil temperature in the inner and outer thawing regions is $T_{\mathrm{u}}$, soil temperature in the frozen region is $T_{f}$, and all the temperatures are only related to time $(t)$ and coordinate $(x)$. The thawing front is a moving phase transition boundary, its distance from the inner or outer edge of the initial frozen wall is $X(t)$, and the temperature $\left(T_{\mathrm{r}}\right)$ at the thawing front is $0^{\circ} \mathrm{C}$ during the process of frozen wall thawing from outside to inside.

The differential equations of heat conduction in thawing region and frozen region are as follows:

$\frac{\partial T_{\mathrm{u}}}{\partial t}=\alpha_{\mathrm{u}} \frac{\partial^{2} T_{\mathrm{u}}}{\partial x^{2}}, \quad(0 \leq x \leq X(t))$

$\frac{\partial T_{\mathrm{f}}}{\partial t}=\alpha_{\mathrm{f}} \frac{\partial^{2} T_{\mathrm{f}}}{\partial x^{2}}, \quad(X(t) \leq x \leq \infty)$

where $a_{\mathrm{f}}$ and $a_{\mathrm{u}}$ are the thermal diffusivity of frozen soil and thawed soil.

$\alpha_{f}=\frac{k_{f}}{c_{f} \rho_{f}}, \alpha_{u}=\frac{k_{u}}{c_{u} \rho_{u}}$

where $k_{\mathrm{f}}$ and $k_{\mathrm{u}}$ are the thermal conductivity of frozen soil and thawed soil, respectively; $c_{\mathrm{f}}$ and $c_{\mathrm{u}}$ are the specific heat of frozen soil and thawed soil, respectively; and $\rho_{\mathrm{f}}$ and $\rho_{\mathrm{u}}$ are the saturated density of frozen soil and thawed soil.

The initial uniform temperature of the frozen wall is equal to the average temperature of the frozen wall $\left(T_{\mathrm{v}}\right)$ without considering the temperature gradient in the frozen wall; thus, the initial condition of the differential equation is as follows:

$T_{f}=T_{u}=T_{v},(t=0)$

The boundary condition of the differential equation is as follows:

$\left\{\begin{array}{lr}T_{u}=T_{b} & (x=0) \\ T_{f}=T_{u}=T_{r}=0 & (x=X(t)) \\ T_{f}=T_{v} & (x=\infty)\end{array}\right.$

The heat balance equation at the inner and outer thawing front $(X(t))$ is as follows:

$\left(k_{f} \frac{\partial T_{f}}{\partial x}-k_{u} \frac{\partial T_{u}}{\partial x}\right)_{x=X(t)}=L \frac{d X(t)}{d t}$

where $L$ is the latent heat of phase change per unit volume of soil.

Therefore, the temperature distributions in the inner and outer thawing region and frozen region are as follows:

$T_{u}=T_{b}\left[1-\frac{\Phi\left(\frac{x}{2 \sqrt{\alpha_{u} t}}\right)}{\Phi\left(\frac{B}{2 \sqrt{\alpha_{u}}}\right)}\right] \quad(0 \leq x \leq X(t))$

$T_{f}=T_{v}\left[1-\frac{\Phi\left(\frac{x}{2 \sqrt{\alpha_{f} t}}\right)}{\Phi\left(\frac{B}{2 \sqrt{\alpha_{f}}}\right)}\right] \quad(X(t) \leq x \leq \infty)$

where $\Phi(y)$ is a Gaussian error function, and it can be shown as follows:

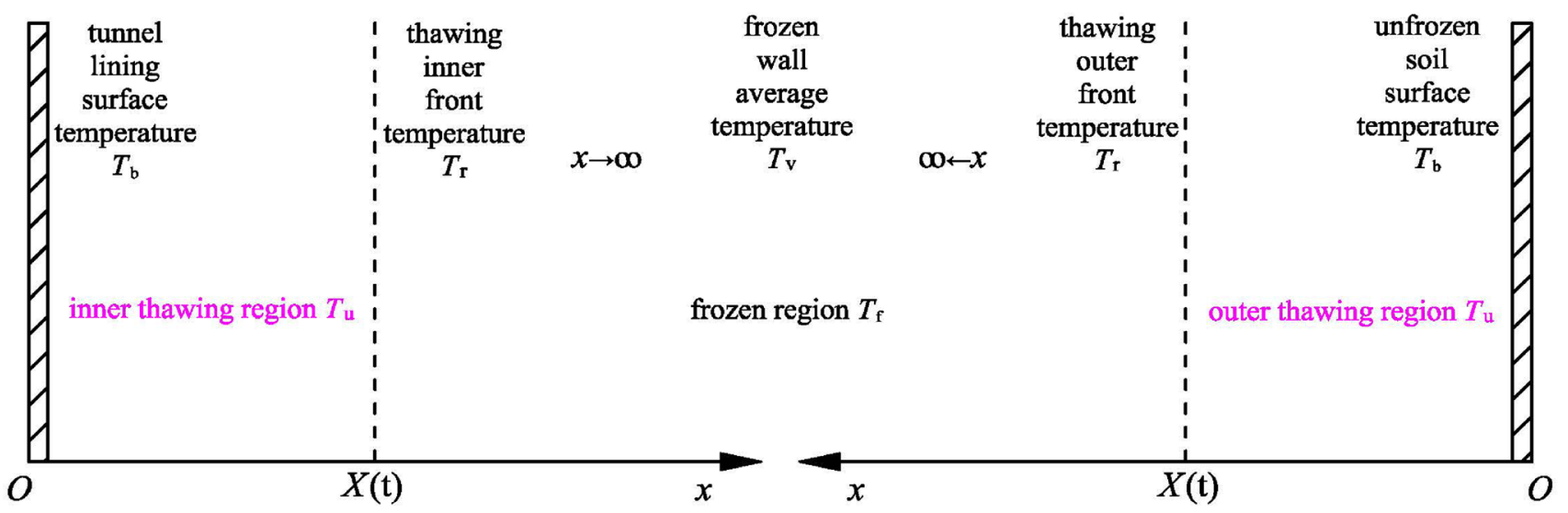

Fig. 5 Schematic diagram of plate thawing theory 
$\Phi(y)=\frac{2}{\sqrt{\pi}} \int_{0}^{y} e^{-\eta^{2}} d \eta$

When $x=X(t), T_{\mathrm{f}}=T_{\mathrm{u}}=T_{\mathrm{r}}=0$, it can be found that the thawing front $(X(t))$ is proportional to the square root of thawing time, namely:

$X(t)=B \sqrt{t}$

where $B$ is the undetermined constant, satisfying the following equation:

$\frac{k_{u} T_{b} e^{-\frac{B^{2}}{4 \alpha_{u}}}}{\sqrt{\alpha_{u}} \Phi\left(\frac{B}{2 \sqrt{\alpha_{u}}}\right)}+\frac{k_{f} T_{v} e^{-\frac{B^{2}}{4 \alpha_{f}}}}{\sqrt{\alpha_{f}}\left[1-\Phi\left(\frac{B}{2 \sqrt{\alpha_{f}}}\right)\right]}=L B \frac{\sqrt{\pi}}{2}$

The thawing front $(X(t))$ can be calculated by Eq. (37) after determining the thermal conductivity, specific heat and density of frozen soil and thawed soil. The initial outer radius of frozen wall is $R_{1}$, and the initial inner radius is $R_{0}$; correspondingly, the radius of the inner and outer thawing front of frozen wall can be obtained as follows:

$R_{1}(t)=R_{0}+B \sqrt{t}$

$R_{2}(t)=R_{1}-B \sqrt{t}$

It should be noted that the thawing speed of the inner and outer edges of the frozen wall is different, and the thawing speed of the inner edge of the frozen wall is greater than that of the outer edge $[27,28]$ under natural thawing conditions in actual engineering. There are many factors affecting the thawing speed of frozen soil, such as the heat exchange capacity [29] and the unfrozen water content [30] of frozen soil, ambient temperature [31] and ventilation [32]. For the tunnel construction using artificial ground freezing technique, the ambient temperature and ventilation around the excavation surface are the main reasons for the different thawing speeds of the inner and outer edges of the frozen wall. In this paper, it is considered to be synchronous due to the lack of quantitative research.

\section{Determination of inner radius of thawing shrinkage region and consolidation region}

Цытович [4] proposed the calculation equation of stable settlement of frozen soil thawing based on the indoor compression test of natural frozen soil:

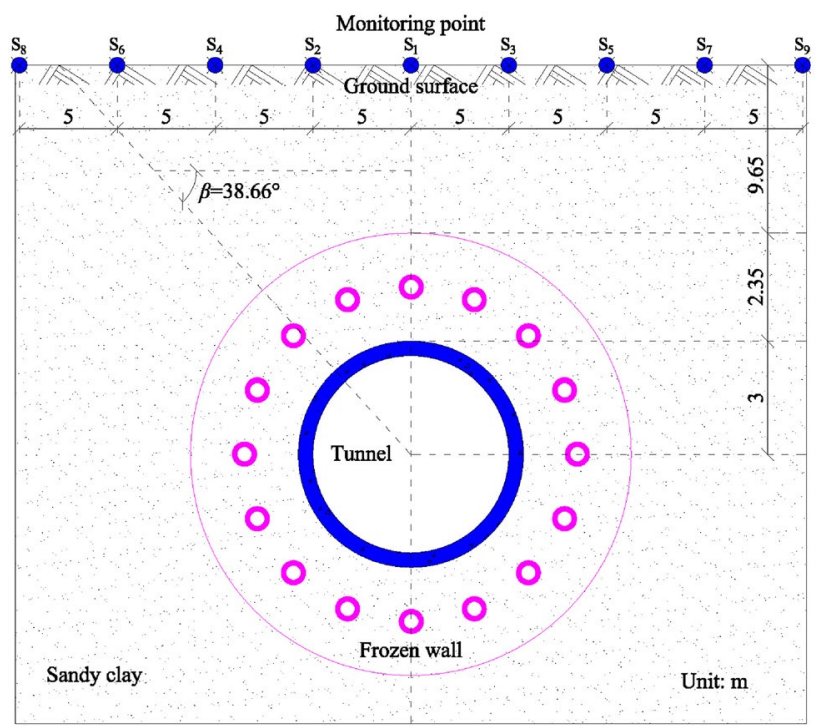

Fig. 6 Case calculation model diagram

$S=\varepsilon_{t h} h_{r}+\varepsilon_{a} p h_{r}$

where $S$ is the stable settlement of the thawed soil; $h_{r}$ is the thickness of thawed soil; $p$ is the external load of thawed soil; $\varepsilon_{\text {th }}$ is the thawing settlement coefficient of frozen soil; and $\varepsilon_{\mathrm{a}}$ is the compaction coefficient of thawed soil.

The external load $(p)$ is approximately considered as the self-weight of the overlying soil of the tunnel without considering the ground surface overload.

$p=\gamma h$

where $\gamma$ is the average volumetric weight of each soil layer over the tunnel; $h$ is the distance between the tunnel center and the ground surface.

The vertical settlement of soil can be considered as the sum of the settlement of all micro units in the region, so the inner radius of thawing shrinkage region of the inner edge of frozen wall is:

$R_{a}(t)=R_{1}(t)-\int_{R_{0}}^{R_{1}(t)} \varepsilon_{t h} d r$

The inner radius of consolidation region of the inner edge of frozen wall is:

$R_{b}(t)=R_{a}(t)-\int_{R_{0}}^{R_{a}(t)} \varepsilon_{\mathrm{a}} \gamma h d r$

The inner radius of thawing shrinkage region of the outer edge of frozen wall is: 
Table 1 Structural dimension parameters of tunnel

\begin{tabular}{llll}
\hline $\begin{array}{l}\text { Tunnel } \\
\text { depth/m }\end{array}$ & $\begin{array}{l}\text { Outer radius of } \\
\text { tunnel lining/m }\end{array}$ & $\begin{array}{l}\text { Outer radius of } \\
\text { freezing pipe/ } \\
\mathrm{mm}\end{array}$ & $\begin{array}{l}\text { Effective } \\
\text { thickness of } \\
\text { frozen wall/m }\end{array}$ \\
\hline 15 & 3 & 80 & 2.35 \\
\hline
\end{tabular}

$R_{c}(t)=R_{1}-\int_{R_{2}(t)}^{R_{1}} \varepsilon_{t h} d r$

The inner radius of consolidation region of the outer edge of frozen wall is:

$R_{d}(t)=R_{c}(t)-\int_{R_{2}(t)}^{R_{c}(t)} \varepsilon_{a} \gamma h d r$

\section{Case analysis}

Figure 6 shows a full section tunnel construction project using artificial ground freezing technology (AGF) [33-35]. The geological survey report shows that the soil property of the tunnel construction stratum is sandy clay, its cohesion (c) is $0 \mathrm{kPa}$, and internal friction angle is $12.68^{\circ}$. According to Eq. (17), the main influence angle $(\beta)$ is $38.66^{\circ}$; correspondingly, the tangent value of main influence angle is approximately 0.8 . In addition, the structural dimension parameters of tunnel are shown in Table 1, the physical structural parameters of soil are shown in Table 2, and the thermophysical parameters of thawed and frozen soil are shown in Table 3.

Besides, the ground surface monitoring distance is approximately set as $20 \mathrm{~m}$ on the left and right bounded by the tunnel centerline, whose computing method comes from the equation of $D=h / \tan \beta$, and this distance also be used as the calculation range of theoretical prediction model. A total of nine ground surface displacement monitoring points $\left(\mathrm{S}_{1} \sim \mathrm{S}_{9}\right)$ are set in this case, and the space between adjacent two points is $5 \mathrm{~m}$.

The thawing front $(X(t))$ can be obtained by substituting $" T_{b}=15^{\circ} \mathrm{C}, "$ " $T_{\mathrm{v}}=--10^{\circ} \mathrm{C}$," and the thermophysical parameters into Eqs.(36) and (37):

$X(t)=127.8 \sqrt{t} \mathrm{~mm}$

The time $\left(t_{j}\right)$ of the horizontal frozen wall completed natural thawing is as follows:

$t_{j}=\left(\frac{2350}{2 \times 127.8}\right)^{2}=85 d$

The inner thawing front radius of frozen wall is as follows according to Eq. (38):

$R_{1}(t)=3000+127.8 \sqrt{t} \mathrm{~mm}$

The inner radius of thawing shrinkage region of the inner edge of frozen wall is as follows according to Eq. (42):

$R_{a}(t)=3000+0.99 \times 127.8 \sqrt{t} \mathrm{~mm}$

The inner radius of consolidation region of the inner edge of frozen wall is as follows according to Eq. (43):

$R_{b}(t)=3000+0.9603 \times 127.8 \sqrt{t} \mathrm{~mm}$

The outer thawing front radius of frozen wall is as follows according to Eq. (39):

$R_{2}(t)=5350-127.8 \sqrt{t} \mathrm{~mm}$

The inner radius of thawing shrinkage region of the outer edge of frozen wall is as follows according to Eq. (44):

$R_{c}(t)=5350-0.01 \times 127.8 \sqrt{t} \mathrm{~mm}$

The inner radius of consolidation region of the outer edge of frozen wall is as follows according to Eq. (43):

Table 2 Physical structural parameters of soil

\begin{tabular}{lllllll}
\hline Soil & $\begin{array}{l}\text { Permeability coef- } \\
\text { ficient/mm·d }\end{array}$ & Void ratio & $\begin{array}{l}\text { Volumetric weight of } \\
\text { water } / \mathrm{N} \cdot \mathrm{mm}^{-3}\end{array}$ & $\begin{array}{l}\text { Average volumetric } \\
\text { weight of soil/ } / \mathrm{mm}^{-3}\end{array}$ & $\begin{array}{l}\text { Thawing settlement } \\
\text { coefficient }\end{array}$ & $\begin{array}{l}\text { Compaction coef- } \\
\text { ficient/MPa }\end{array}$ \\
\hline Sandy clay & 2.592 & 0.76 & $1 \mathrm{e}^{-5}$ & $1.93 \mathrm{e}^{-5}$ & 0.01 & 0.01 \\
\hline
\end{tabular}

Table 3 Thermophysical parameters of soil

\begin{tabular}{lllll}
\hline Soil & $\begin{array}{l}\text { Saturation density/ } \\
\mathrm{g} \cdot \mathrm{cm}^{-3}\end{array}$ & $\begin{array}{l}\text { Thermal conductivity// } \\
\mathrm{kcal} \cdot\left(\mathrm{m} \cdot{ }^{\circ} \mathrm{C} \cdot \mathrm{d}\right)^{-1}\end{array}$ & $\begin{array}{l}\text { Specific heat/ } \\
\mathrm{kcal} \cdot\left(\mathrm{kg} \cdot{ }^{\circ} \mathrm{C}\right)^{-1}\end{array}$ & $\begin{array}{l}\text { Latent heat/ } \\
\mathrm{kcal} \cdot \mathrm{m}^{-3}\end{array}$ \\
\hline Frozen & 1.928 & 32.46 & 0.27 & $24,421.28$ \\
Unfrozen & & 23.14 & 0.34 & \\
\hline
\end{tabular}




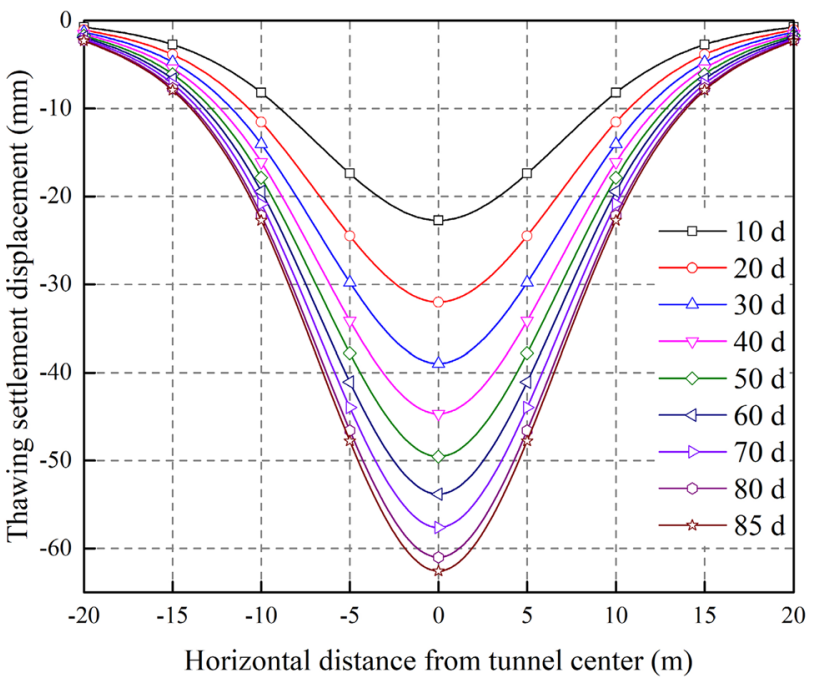

Fig. 7 Predictive curve of the ground surface thawing settlement

$R_{d}(t)=5350-0.0397 \times 127.8 \sqrt{t} \mathrm{~mm}$

The calculation program of thawing settlement under natural thawing condition is compiled by Maple software according to Eq. (20). The law of the ground surface thawing settlement is shown in Fig. 7 during the natural thawing period of horizontal frozen wall as the calculation range is taken as $-20 \mathrm{~m} \leq x \leq 20 \mathrm{~m}$.

Figure 7 shows that the accumulated thawing settlement displacement of the ground surface gradually decreases with the increase in the horizontal distance from tunnel center during the natural thawing period. The accumulated thawing settlement displacement of ground surface at tunnel center axis is $-22.737 \mathrm{~mm},-32.022 \mathrm{~mm}$, $-38.977 \mathrm{~mm},-44.679 \mathrm{~mm},-49.552 \mathrm{~mm},-53.815 \mathrm{~mm}$, $-57.599 \mathrm{~mm},-60.991 \mathrm{~mm}$ and $-62.561 \mathrm{~mm}$ after thawing for $10 d, 20 d, 30 d, 40 d, 50 d, 60 d, 70 d, 80 d$ and $85 d$, respectively, while it is only $-2.305 \mathrm{~mm}$ at $\pm 20 \mathrm{~m}$ away from tunnel centerline after thawing for $85 \mathrm{~d}$, which indicates that the influence distance of the ground surface thawing settlement is less than $\pm 6.7 R_{0}$ when the horizontal frozen wall completely natural thaws.

Cai et al. [9] also predicted the thawing settlement displacement of the same project, but there was no obvious distinction between the thawing stage and the consolidation stage in their prediction model. In order to reflect the optimization degree of this model in these two stages, the ground surface settlement law of these two models is shown in Fig. 8.

Figure 8a shows that the variation trend of the ground surface thawing settlement in this model is as same as Cai's model [9], but the predicted value of this model is generally larger than that of Cai's model. Compared with the field measured data, the predicted value of Cai's model is conservative, while the predicted value of this model is closer to the measured data. Specifically, the accumulated thawing settlement displacement in this model is $-62.561 \mathrm{~mm}$ at the tunnel centerline, while it is $-40.700 \mathrm{~mm}$ in Cai's model; subtracting from the field measured value, it is with the difference of $7.610 \mathrm{~mm}$ and $14.700 \mathrm{~mm}$, respectively. It also shows that the displacement caused by the consolidation of thawed soil cannot be ignored, and this model has the advantage to describe this stage.

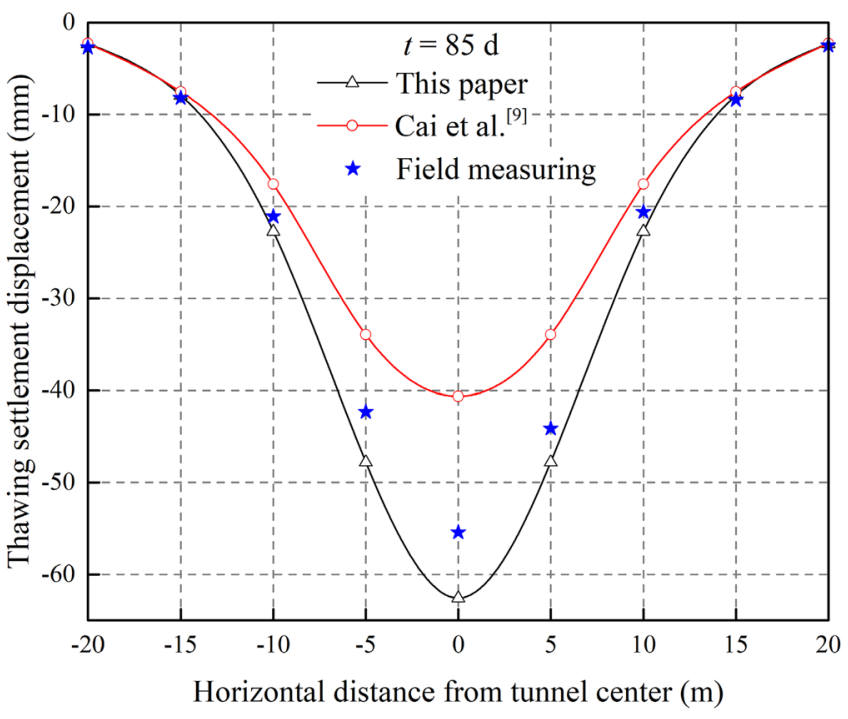

(a)

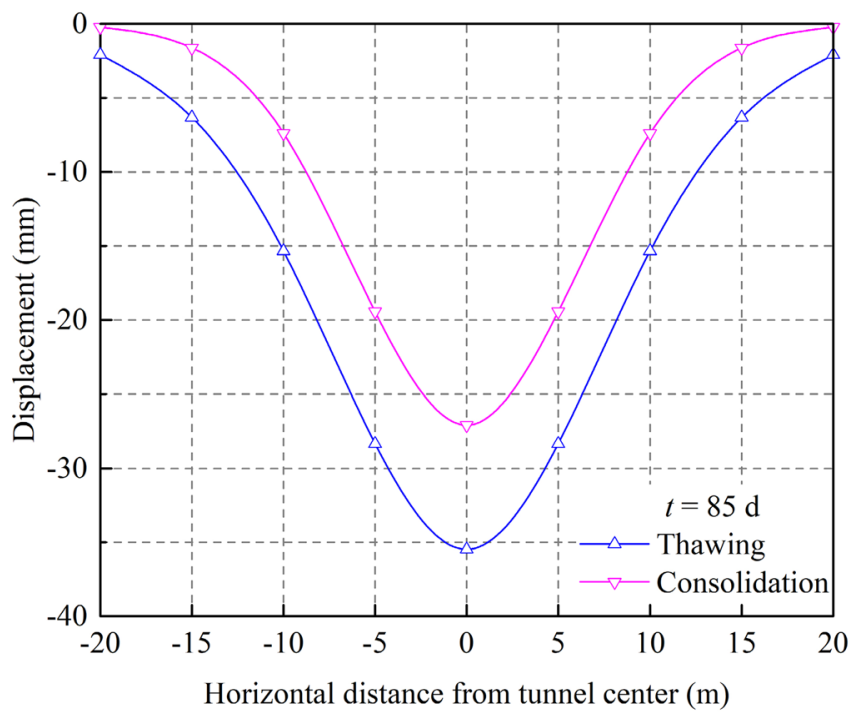

(b)

Fig. 8 Predictive curve of ground surface settlement: a comparison of Cai's model and this model; $\mathbf{b}$ comparison of thawing displacement and consolidation displacement 
In addition, Fig. $8 \mathrm{~b}$ shows that the displacement generated by the consolidation of thawed soil is less than that generated by the thawing of frozen soil in this model, and the consolidation of thawed soil has hysteresis and timeliness. Specifically, the consolidation displacement is $-27.084 \mathrm{~mm}$ at the tunnel centerline, while the thawing displacement is $-35.476 \mathrm{~mm}$, with the difference of $7.392 \mathrm{~mm}$. The thawing displacement is dominant in the early stage of thawing, while the consolidation displacement begins to generate after the thawed soil existence, which reflects the nonsimultaneity of these two displacements. Besides, the consolidation of soil will continue to develop with time after the horizontal frozen wall completely thawing, which is consistent with the conclusion of Wang et al. [36].

The accumulated thawing settlement displacement of tunnel horizontal frozen wall during thawing period is the key factor to judge whether the project site needs to adopt compaction grouting reinforcement, and the ground surface thawing settlement velocity is the key parameter to determine the grouting reinforcement time. Figure 9 reflects the diachronic change law of the ground surface thawing settlement.

Figure 9 shows that the thawing settlement velocity of each point of the ground surface is first fast and then slow during the natural thawing period of horizontal freezing wall. For example, the thawing settlement velocity of the ground surface is $2.274 \mathrm{~mm} / \mathrm{d}, 0.928 \mathrm{~mm} / \mathrm{d}, 0.696 \mathrm{~mm} / \mathrm{d}$, $0.570 \mathrm{~mm} / \mathrm{d}, 0.487 \mathrm{~mm} / \mathrm{d}, 0.426 \mathrm{~mm} / \mathrm{d}, 0.378 \mathrm{~mm} / \mathrm{d}$, $0.339 \mathrm{~mm} / \mathrm{d}$ and $0.157 \mathrm{~mm} / \mathrm{d}$ after thawing for $10 \mathrm{~d}$, 20d, 30d, 40d, 50d, 60d, 70d, 80d and 85d, respectively. The ground surface has experienced a certain degree of frost heaving deformation in the positive freezing period, so the ground surface has a rapid thawing settlement

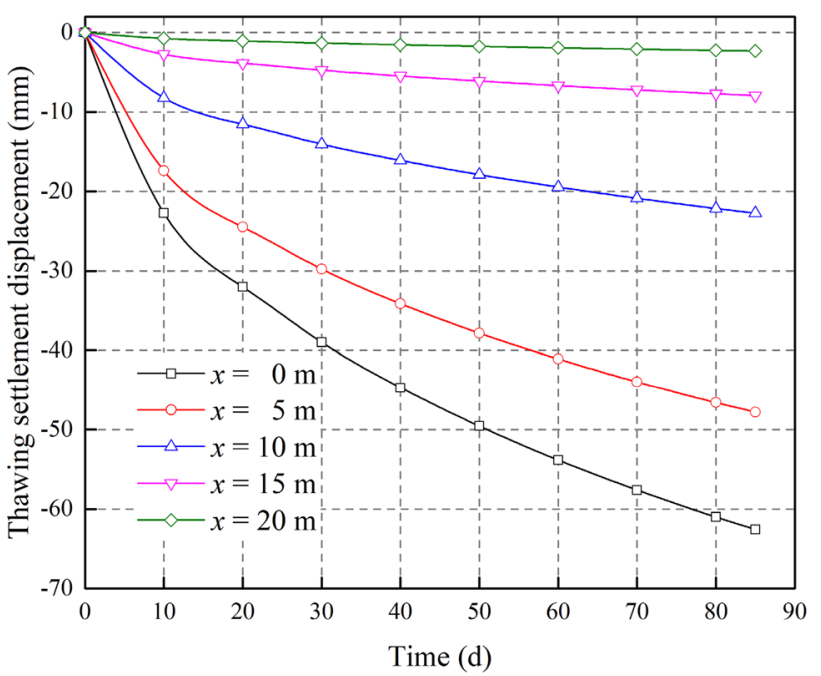

Fig. 9 Diachronic change curve of the ground surface thawing settlement deformation after thawing for $10 \mathrm{~d}$, which is theoretically considered to be the process of surface displacement clearing, and this stage is considered as the safety period of engineering protection. Besides, the thawing settlement velocity of each point of the ground surface gradually tends to be stable after thawing for $30 \mathrm{~d}$. Therefore, in view of the large deformation of the ground surface during the natural thawing period in the project, it is theoretically considered that thawing for $20 \sim 30 \mathrm{~d}$ is the best grouting time.

According to the distribution of ground surface frost heaving, the location of grouting holes shall be reasonably arranged. Besides, it is required to closely monitor the temperature change of temperature measuring holes, and adopt the interval grouting method to ensure the unity of grouting amount and thawing settlement. The grouting pressure is generally set as 2 to 2.5 times of the hydrostatic pressure, and the grouting volume is usually 1.15 times [37] that of frozen soil.

In order to further explain the influence of the thawing of frozen soil and the consolidation of thawed soil on the speed of ground surface settlement, the comparison of the diachronic thawing settlement of ground surface at the tunnel centerline is shown in Fig. 10.

Figure 10a shows that the thawing settlement velocity of this model is significantly higher than that of Cai's model [9] at the tunnel centerline. For example, the thawing settlement velocity of this model is $2.274 \mathrm{~mm} / \mathrm{d}$ after natural thawing for $10 \mathrm{~d}$, while it is $1.390 \mathrm{~mm} / \mathrm{d}$ of Cai's model. There is a certain gap between them and the velocity of the field measured data $(2.05 \mathrm{~mm} / \mathrm{d})$, but the velocity is closer to this model. In addition, Fig. 10b shows that the curvature of thawing displacement and consolidation displacement is almost the same within 20 days of natural thawing at the tunnel centerline. Besides, the consolidation displacement is obviously smaller than the thawing displacement after natural thawing for $20 \mathrm{~d}$, and the change rate of consolidation displacement is slow, with an average value of $0.173 \mathrm{~mm} / \mathrm{d}$, while the change rate of thawing displacement is fast, with an average value of $0.298 \mathrm{~mm} / \mathrm{d}$. The thawing displacement will finish with the horizontal frozen wall completely thawing, while the consolidation displacement will continue at a lower settlement rate for a long time (Table 4). Therefore, the accumulated thawing settlement displacement of the stratum is further increased after considering the consolidation of the thawed soil, which is helpful for the project site to predict in advance and take the compaction grouting measures to control the thawing settlement. 


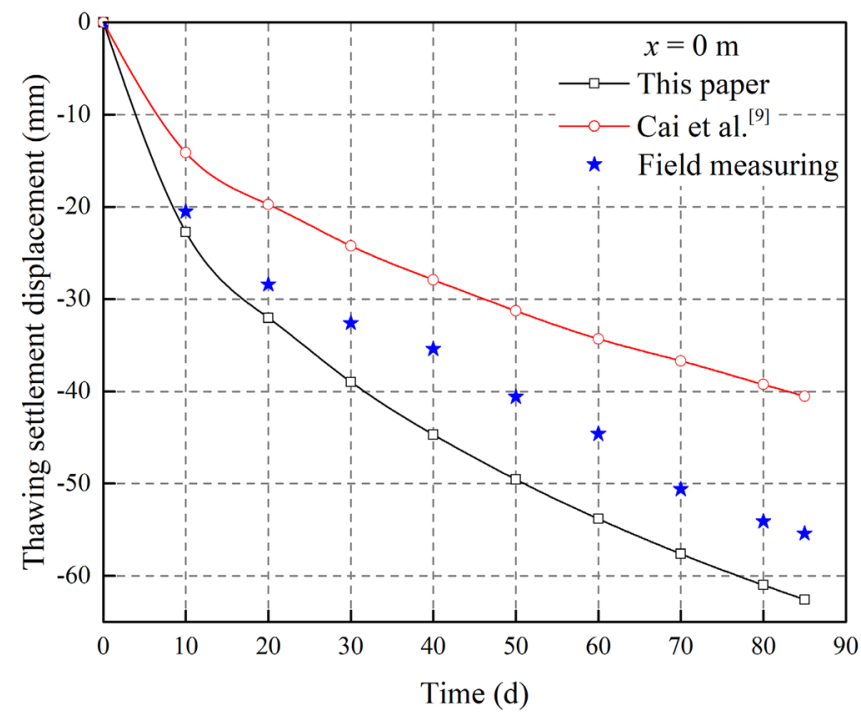

(a)

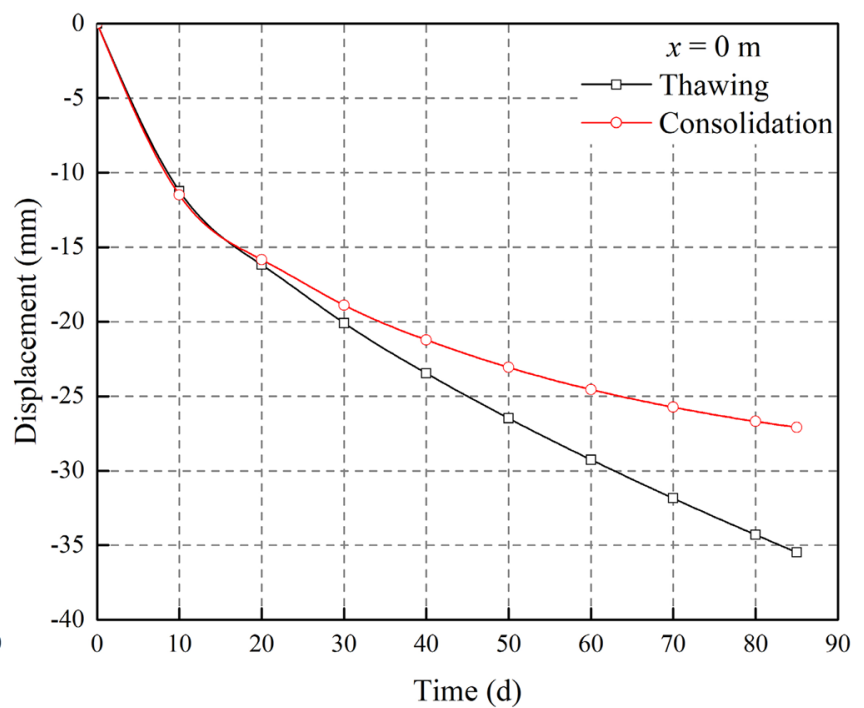

(b)

Fig. 10 Diachronic change curve of ground surface settlement: a comparison of Cai's model and this model; b comparison of thawing displacement and consolidation displacement

Table 4 Gauss-Legendre integral point

\begin{tabular}{lll}
\hline$N$ & $x_{k}$ & $A_{k}$ \\
\hline 1 & \pm 0.5773503 & 1 \\
2 & \pm 0.7745967 & 0.5555556 \\
& 0 & 0.8888889 \\
3 & \pm 0.8611363 & 0.3478548 \\
& \pm 0.3399810 & 0.6521452 \\
4 & \pm 0.9061798 & 0.2369269 \\
& \pm 0.5384693 & 0.4786287 \\
& 0 & 0.5688889 \\
5 & \pm 0.9324695 & 0.1713245 \\
& \pm 0.6612094 & 0.3607616 \\
& \pm 0.2386198 & 0.4679139 \\
\hline
\end{tabular}

\section{Conclusion}

Based on the research results of Cai et al., a diachronic prediction model of ground surface thawing settlement is established by combining consolidation compaction function with stochastic medium theory considering the thawing of frozen soil and the consolidation of thawed soil during the thawing period of tunnel horizontal frozen wall. Specifically, based on the plate freezing theory, the calculation equations of the inner and outer radius of thawing shrinkage region and consolidation region are established considering the thawing settlement coefficient and compaction coefficient.

(1) The case analysis results show that, firstly, the accumulated thawing settlement gradually decreases with the horizontal distance from the tunnel center gradually increasing during the natural thawing period. The accumulated thawing settlement increases rapidly at the initial stage of thawing, while the accumulated thawing settlement decreases gradually with the extension of thawing time. Secondly, the comparison curve of the thawing displacement and consolidation displacement shows that the thawing displacement of ground surface is greater than the consolidation displacement. The thawing displacement will finish with the horizontal frozen wall completely thawing, while the consolidation displacement will continue at a lower settlement rate for a long time. Finally, the thawing settlement predicted in this paper is larger than that of Cai et al., which indicates that the consolidation settlement in natural thawing period cannot be ignored.

(2) It is suggested that this diachronic prediction method should be adopted in advance to predict the thawing settlement of the ground surface during the natural thawing period of horizontal frozen wall in the design stage of tunnel freezing engineering, and then taking the appropriate compaction grouting measures to reduce the impact of ground surface thawing settlement on the surrounding environment.

\section{Data Availability and material}

The datasets generated and analyzed during the current study are available from the corresponding author upon reasonable request. 
Acknowledgements This research was supported by National Natural Science Foundation of China (Grant No. 51778004), Research Activities Funding for Reserve Candidate of Academic and Technical Leaders of Anhui Province, China (Grant No. 2018H170) and Academic Funding for Top-notch Talents in University Disciplines (Majors) of Anhui Province, China (Grant No. gxbjZD10). In addition, Rongbao Hong is responsible for the numerical simulation and thesis writing; Haibing Cai is responsible for the derivation of theoretical equations; and Mengkai $\mathrm{Li}$ is responsible for data processing. Moreover, thanks for the data support in the literature written by Cai et al. and its DOI is https://doi.org/10.16285/j.rsm.2014.02.029.

\section{Declarations}

Conflicts of Interest The authors declare that there are no conflicts of interest regarding the publication of this paper.

Open Access This article is licensed under a Creative Commons Attribution 4.0 International License, which permits use, sharing, adaptation, distribution and reproduction in any medium or format, as long as you give appropriate credit to the original author(s) and the source, provide a link to the Creative Commons licence, and indicate if changes were made. The images or other third party material in this article are included in the article's Creative Commons licence, unless indicated otherwise in a credit line to the material. If material is not included in the article's Creative Commons licence and your intended use is not permitted by statutory regulation or exceeds the permitted use, you will need to obtain permission directly from the copyright holder. To view a copy of this licence, visit http://creativecommons. org/licenses/by/4.0/.

\section{References}

1. Chen XS (1996) 40 years of ground freezing technology. Coal Sci Tech. 1:13-15. https://doi.org/10.13199/j.cst.1996.01.13. chenxsh.004

2. Cheng $H(2000)$ Present situation and prospect of artificial ground freezing technology in urban underground engineering. J Huainan Min Inst 2:17-22

3. He MY (2019) Technical research on artificial horizontal freezing method in subway construction. Xi'an Univ Sci Tech, Xi'an, China

4. Цытович НА (1985) Frozen soil mechanics. Sci Press, Beijing

5. Orlando BA, Branko L (2011) Permafrost engineering. China Cons Ind Press, Beijing

6. Tao XL, Ma JR, Huang L (2015) Thaw settlement test of artificial frozen soil and prediction of thaw settlement coefficient. J Min Safety Eng. 6:996-100. https://doi.org/10.13545/j.cnki. jmse.2015.06.021

7. Yang FX, Zhang XF, Leng YF, Zhao YM (2011) Empirical determination method of volumetric compression coefficient of frozen soil thawing. Rock Soil Mech. 32(11):3432-3436. https:// doi.org/10.16285/j.rsm.2011.11.052

8. Nixon N (1971) One-dimensional consolidation of thawing soils. Can Geotech J 8(4):565-588. https://doi.org/10.1139/ t71-057

9. Cai HB, Peng LM, Zheng TL (2014) Duration prediction model of surface thaw settlement during horizontal freezing construction of tunnel. Rock Soil Mech. 35(2):504-510. https:// doi.org/10.16285/j.rsm.2014.02.029

10. Ghiasi V, Koushki M (2020) Numerical and artificial neural network analyses of ground surface settlement of tunnel in saturated soil. SN Appl Sci 2(5):1-14. https://doi.org/10.1007/ s42452-020-2742-z

11. Mikaeil R, Beigmohammadi M, Bakhtavar E, Haghshenas SS (2019) Assessment of risks of tunneling project in Iran using artificial bee colony algorithm. SN Appl Sci 1(12):1-9. https:// doi.org/10.1007/s42452-019-1749-9

12. Zhang Q, Wu K, Cui S, Yu Y, Zhang Z, Zhao J (2019) Surface settlement induced by subway tunnel construction based on modified Peck formula. Geotech Geol Eng 37:2823-2835. https://doi.org/10.1007/s10706-018-00798-6

13. Wei G, Zhang XH, Xu YF, Wang Z (2019) Prediction of ground settlement due to excavation of a Quasi-Rectangular shield tunnel based on stochastic medium theory. Geotech Geol Eng 37:3605-3618. https://doi.org/10.1007/s10706-019-00856-7

14. Yang JS, Liu BC (2002) Ground movement and deformation caused by urban tunnel construction. China Ry Press, Beijing

15. Ning FB (2005) Analysis of the influence of soil frost heaving and thawing settlement on surface deformation in underground freezing engineering. Gen Inst coal sci, Beijing, China

16. Liu B, Li DY, Liao JJ (2011) The effect of earth pressure on ground settlement after frozen soil thawing in connect aisle construction of metro engineering. J China Coal Soc. 4:551555. https://doi.org/10.13225/j.cnki.jccs.2011.04.005

17. Shi CH, Peng LM, Zhao D (2008) Calculation of ground deformation caused by tunnel excavation and precipitation considering space-time effect. China Civ Eng J 12:91-96

18. Shi CH, Huang LC (2005) Calculation of soil deformation in disturbed area of pipe jacking construction tunnel. J Central South Univ: (Sci Tech) 2:323-328

19. Cai HB, Peng LM, Zheng TL (2015) Prediction method for surface settlement of horizontal frozen wall during forced thawing period. Rock Soil Mech. 12:3516-3522. https://doi.org/10. 16285/j.rsm.2015.12.023

20. Cai HB. Prediction method and engineering application of ground frost heaving and thawing settlement of subway tunnel horizontal freezing engineering. Central South University, Hunan, China

21. Cai HB, Liu Z, Li S, Zheng TL (2019) Improved analytical prediction of ground frost heave during tunnel construction using artificial ground freezing technique. Tunn Undergr Sp Tech. 92:103050.1-103050.9. https://doi.org/10.1016/j.tust.2019. 103050

22. Cai HB, Huang YC, Li Y (2015) Soil thaw settlement prediction and parameter sensitivity analysis based on stochastic medium theory. Ry Std Dgn. 59(8):107-111. https://doi.org/ 10.13238/j.issn.1004-2954.2015.08.023

23. Cai HB, Peng LM, Zheng TL (2014) Prediction method of ground frost heave in tunnel freezing period based on stochastic medium theory. J Central South Univ: (Sci Tech) 45(12):4251-4257

24. Wu ZZ (2010) Random medium prediction model of tunnel surface deformation based on non-uniform convergence model. J Central South Univ: (Sci Tech) 41(5):2005-2010

25. Li T, Wang YB, Yu ZW, Liu B (2020) Prediction of surface soil movement and settlement during excavation of variable cross-section tunnel. J Central South Univ: (Sci Tech) 51(2):433-444

26. Shi B, Zhu C (2011) Discussion on arithmetic of main influence angle of stratum for prediction of ground settlement. Water Res Hydropower Eng. 42(3):13-15. https://doi.org/10.13928/j. cnki.wrahe.2011.03.001

27. Han YF, Li FZ (2009) Study of frozen soil melting law measuring in the subway contact channels. Shanghai Const Sci Tech 4:53-55 
28. Cai HB, Cheng H, Peng LM, Yao ZS, Rong CX (2009) Model test on displacement field of double-route metro constructed with horizontal freezing method. Chinese J Rock Mech Eng 28(10):2088-2095

29. Hu X, Wu Y, Li X (2019) A field study on the freezing characteristics of freeze-sealing pipe roof used in ultra-shallow buried tunnel. Appl Sci 9(8):1-10. https://doi.org/10.3390/app90 81532

30. Wang D, Wang Y, Ma W, Lei L, Wen Z (2018) Study on the freezing-induced soil moisture redistribution under the applied high pressure. Cold Reg Sci Technol. 145:135-141. https:// doi.org/10.1016/j.coldregions.2017.10.012

31. Fu Y, Hu J, Liu J, Zeng $H$ (2020) Finite element analysis of natural thawing heat transfer of artificial frozen soil in shielddriven tunnelling. Adv Civ Eng 2020:1-18. https://doi.org/10. $1155 / 2020 / 2769064$

32. Kim DY, Lee HS, Sim BK (2011) A study on the design of tunnel lining insulation based on measurement of temperature in tunnel. J Korea Tunn Undergr Sp Assoc 13(4):319-345
33. Cai HB, Peng LM, Zheng TL (2012) A duration prediction model of surface frost heave induced by tunnelling with horizontal freezing method. Rock Soil Mech. 33(6):1761-1768. https:// doi.org/10.16285/j.rsm.2012.06.031

34. Cheng $\mathrm{H}$, Zang $\mathrm{H}$ (2003) Quasi-coupling numerical analysis of frost heave effect of horizontal freezing in artificial ground. Chinese J Geotech Eng 1:87-90

35. Zang $H(2000)$ Numerical analysis of frost heave effect of horizontal ground freezing. Huainan Institute technology, Huainan, China

36. Wang JP, Wang WS, Shi TS (1999) Model test of frost heaving and thawing settlement of artificially frozen soil. J China Univ min tech 4:303-306

37. Cao HL (2010) Thawing settlement control of subway tunnel construction by artificial ground freezing. Chinese J UndergrSp Eng 6(2):387-390

Publisher's Note Springer Nature remains neutral with regard to jurisdictional claims in published maps and institutional affiliations. 